\title{
¿Son necesarios los programas de reducción de daños en el tratamiento de la dependencia alcohólica?
}

\author{
Cuadrado Callejo, P. \\ Psiquiatra. Coordinador del Programa de Problemas Relacionados con el Alcohol. \\ Enviar correspondencia a: \\ Pedro Cuadrado Callejo. SSM Retiro. C/ Doctor Castelo 60. 28009 Madrid. Tfno 915868524. FAX 915868763.
}

\section{Resumen:}

La tendencia a la cronicidad de más de un tercio de los dependientes del alcohol, la estabilidad de los patrones de evolución y la capacidad limitada de los tratamientos para modificar la evolución a largo plazo de este grupo de pacientes, llevan a plantear la necesidad de programas de reducción de daños en el tratamiento de la dependencia alcohólica.

Estos programas estarían indicados, fundamentalmente, en aquellos pacientes con latencias muy cortas de recaída: incapaces de mantenerse abstinentes más de cuatro o seis semanas continuadas a lo largo del tiempo, y vendrían definidos por el cambio de prescripción y de objetivo desde la abtinencia absoluta a la reducción del consumo y por la adaptación de los dispositivos asistenciales para intervenir prioritariamente sobre las consecuencias del consumo.

\section{Summary:}

The tendency towards chronicity of more than one third of alcohol dependants, the stability of evolution patterns and the limited ability of treatments to modify long-term evolution on this group of patients, lead us to express the need of harm reduction programs in alcohol dependence.

These programs would be mainly advised in patients with short latency relapse: those who are unable to remain abstinent for more than four or six weeks without interruption. These programs would be defined by the change of the objective from complete abstinence to drink reduction and by the adjustment of welfare services to take part, first and foremost, in consumption consequences.

Key words: ALCOHOL DEPENDENCE. ALCOHOLISM. TREATMENT. HARM REDUCTION.

Palabras clave: DEPENDENCIA ALCOHOLICA. ALCOHOLISMO. TRATAMIENTO. REDUCCION DE DAÑOS.

\section{INTRODUCCION}

$\mathbf{E}$ I concepto de disminución de daños y riesgos se comienza a utilizar con amplitud en el mundo de las adicciones a finales de los 80 , sobre todo como respuesta a las consecuencias de la infección por VIH en usuarios de drogas por vía parenteral. La gravedad del SIDA hace que su control se haga prioritario y relegue a un segundo término el control de la enfermedad adictiva. Si se llega a la necesidad de optar entre el control de la enfermedad adictiva y el control de la complicaciones del consumo, en este caso el SIDA, es porque las estrategias que existían para el control de la enfermedad adictiva, los programas libres de drogas, no solo no conseguían la abstinencia ni reducían el consumo en un gran porcentaje de pacientes, sino que además alejaban a éstos de los dispositivos sanitarios. El objetivo básico de los programas de reducción de daños es conseguir el acerca- miento a los dispositivos sociosanitarios como punto de partida para disminuir la mortalidad, morbilidad y mejorar la calidad de vida de estos pacientes (1-3).

Estos programas surgen inicialmente dirigidos a un grupo de pacientes restringidos ( HIV, embarazadas, enfermedades severas asociadas ) y con el tiempo han ido flexibilizando sus criterios y de ser programas dirigidos a solucionar complicaciones de los adictos, pasan al arsenal terapéutico de la propia dependencia, estableciéndose su uso como una alternativa para aquellos dependientes crónicos con múltiples recaídas donde los resultados de los programas libres de drogas no han sido eficaces.

En este sentido, desde el punto de vista del tratamiento de la dependencia de sustancias, son la cronicidad de ésta y los pobres resultados del tratamiento los que avalan el paso desde los programas libres de drogas a los programas de mantenimiento donde limi- 
tar los daños y riesgos del consumo son prioritarios frente a resolver la relación con el consumo.

\section{DE LA CRONICIDAD DE LA DEPENDENCIA ALCOHOLICA}

La evolución de la dependencia alcohólica no es homogénea: un grupo de pacientes remite y un porcentaje alto permanece a lo largo del tiempo con un patrón de consumo y de complicaciones estable (4).

En estudios comunitarios, Ojesjo (5), estudiando la muestra Lundby Cohort a los 15 años de seguimiento, encuentra que un $27 \%$ habían muerto, un $30 \%$ se habían recuperado y un $43 \%$ se mantenían igual o peor que en el momento de la primera evaluación. Vaillant (6), en sus muestras de "colegiales" y Core City, tras 20 a 30 años de seguimiento desde el comienzo de la enfermedad, encuentra tasas de mortalidad de $13 \%$ y $23 \%$ respectivamente, abstinencia estable de $17 \%$ y $29 \%$, abuso crónico de alcohol de $52 \%$ y $33 \%$ y consumo sin problemas de $18 \%$ y $15 \%$.
En la Tabla 1 (7-15), se recogen los estudios más significativos de seguimiento a largo plazo de pacientes que inician tratamiento en un momento dado y que son reevaluados al cabo de 8 a 20 años. Aunque los resultados son diversos por la heterogeneidad de las muestras y porque a veces los criterios de evaluación no coinciden totalmente, se puede decir que entre un tercio y una cuarta parte de los que inician tratamiento siguen estando como estaban tras diez a veinte años de evolución. La combinación de distintos factores que condicionan las tasas de no remisión a largo plazo, como son la edad media de la muestra, las tasas de mortalidad y la tasa de "sin datos", puede explicar las tasas muy bajas de no remitidos de algunos de los estudios reseñados. La disminución de las tasas de dependencia con la edad es un hecho constatado y se debería, como señala Vaillant (6), a un aumento de la mortalidad, que se ha estimado en un $2 \%$ por cada año de seguimiento (13), a una discreta incorporación a la abstinencia estable y a un porcentaje de alrededor del $10 \%$ que reanudan el consumo sin problemas $(16,10,6,17)$.

TABLA 1. Estudios de Seguimiento a largo plazo.

\begin{tabular}{|c|c|c|c|c|c|c|c|}
\hline Autor & Años & $\mathrm{N}$ & Perdidos & Muerte & Abst & Mejoría & No mejoría \\
\hline Lundquist (1973) & 9 & 200 & - & $22.5 \%$ & $28.5 \%$ & $19.5 \%$ & $29.5 \%$ \\
\hline Hyman (1976) & 15 & 54 & $19 \%$ & $33 \%$ & $9 \%$ & $15 \%$ & $24 \%$ \\
\hline Vaillant (1983) & 8 & 106 & $6 \%$ & $27 \%$ & $27 \%$ & $4 \%$ & $36.5 \%$ \\
\hline Edwards y al.(1983) & 13 & 99 & $13 \%$ & $17 \%$ & $32 \%$ & & $38 \%$ \\
\hline O'Connor y Daly (1985) & 20 & 133 & $30 \%$ & $40 \%$ & $20 \%$ & $5 \%$ & $5 \%$ \\
\hline Cross (1990) & 10 & 200 & $21 \%$ & $27 \%$ & $43 \%$ & - & $8.5 \%$ \\
\hline Finney y Moos (1991) & 10 & 113 & $10 \%$ & $17 \%$ & $39 \%$ & $2 \%$ & $32 \%$ \\
\hline Längle (1993) & 10 & 96 & $5 \%$ & $22 \%$ & $51 \%$ & $7 \%$ & $15 \%$ \\
\hline Shaw y al. (1997) & 9 & 112 & $31 \%$ & $15 \%$ & $28 \%$ & $18 \%$ & $8 \%$ \\
\hline
\end{tabular}

\subsection{Estabilidad de los patrones de consumo}

Un hecho también constatado por los estudios de seguimiento a largo plazo es la estabilidad de los patrones de evolución, sobre todo de las tasas de abstinencia. Hay una constancia en las tasas de abstinencia que algunos autores señalan ya desde los primeros seis meses (18); pero que parece mucho más establecido a partir de uno ó dos años de seguimiento $(19,20)$, con tasas de entre el $25 \%$ y el $30 \%$ en los cortes temporales sucesivos, utilizando como período de evaluación el año anterior al corte. Esto es lo que encuentra Vaillant (21) y Längle (14) a los 2, 5 y 10 años. Al mismo tiempo, se encuentra que más de tres cuartas partes de los que estaban abstinentes a los dos o tres años seguían estándolo cinco, ocho y quince años más tarde $(13,6,22)$. La estabilidad es menor en aquellos que siguen consumiendo con pro- blemas, 2/3 de los que tenían este status a los dos años seguían teniéndolo cinco y ocho años más tarde $(13,22)$, y aún menor en aquellos con el status de bebida controlada, donde únicamente un tercio seguían manteniendo este status 15 años más tarde (6).

Por lo tanto, las tasas de abstinencia son bastantes estables en distintos períodos evolutivos, entre un cuarto y un tercio de la muestra, mientras que aquellos con criterios de dependencia van disminuyendo con el tiempo y lo hacen fundamentalmente a costa del aumento de la mortalidad y a un porcentaje pequeño de bebedores que se incorporan al grupo de abstinentes o que mantienen distintos niveles de consumo sin problemas, lo que permite definir a la dependencia alcohólica como una enfermedad, en palabras de Vaillant (6), con una gran estabilidad en los patrones de evolución, con poca progresividad y 
pequeñas fluctuaciones a lo largo del tiempo dentro de un nivel de gravedad conseguido.

\section{DE LOS RESULTADOSY PAPEL DELTRATAMIEN- TO}

La búsqueda de tratamiento en la dependencia alcohólica, casi siempre se hace en situaciones de crisis y para resolver enfermedades y problemas que se atribuyen en mayor o menor medida al consumo de alcohol más que para abordar la dependencia, cuya existencia, características e implicaciones son desconocidas por los pacientes. Esta ausencia de conciencia de enfermedad, explicaría el por que la mitad de los que inician tratamiento se mantienen en éste poco tiempo: así, en una muestra de 161 pacientes en tratamiento ambulatorio, las tasas de abandono pasan desde el $7 \%$ al mes al $27 \%$ a los seis meses, al $37 \%$ al año y al 53\% a los dos años (23). La evolución del consumo y de las complicaciones durante el tiempo que permanecen en tratamiento, suele ser buena a corto plazo y va empeorando progresivamente a lo largo del tiempo: así, en muestras ambulatorias, las tasas de abstinencia pasan del $82 \%$ al mes al $72 \%$ a los tres meses, al $44 \%$ a los seis meses, al $32 \%$ al año y al $28 \%$ a los dos años $(24,25)$.

Estas cifras están dentro del amplio rango encontrado por Emrick (26), en una de las primeras revisiones de eficacia de los tratamientos del alcoholismo, donde sobre 265 estudios, que englobaron a más de trece mil pacientes, publicados en inglés entre 1952 y 1961, encuentra una tasa de abstinencia de 33.8\% y mejoría del consumo y de distintas áreas de funcionamiento del $67.2 \%$. En una revisión más reciente, Monahan y Finney (27), sobre 100 estudios publicados entre 1980 y 1992, con una media de seguimiento de 10.6 meses, encuentran un rango de tasas de abstinencia entre $0 \%$ y $91 \%$ y una media de $43 \%$.

\subsection{Papel del tratamiento}

En las enfermedades crónicas y con recidivas frecuentes, el papel de los tratamientos en la evolución queda desdibujado, sobre todo cuando se alarga el tiempo de seguimiento y se elige como criterio de evaluación la ausencia de enfermedad. Así, Costello (28), en un metaanálisis de estudios de seguimiento de dos años, encuentra que tener trabajo y relaciones afectivas estables predecía la mitad de la varianza de resultados, mientras que al tratamiento solo se le podía atribuir un cuarto de ésta. En esta línea, el estudio Rand, con seguimientos a cuatro años, ponía de manifiesto que las características de los pacientes al entrar en tratamiento correlacionaban más con los resultados que las características de los programas de tratamiento (29), y Moos y Finney (30), en un seguimiento de diez años encuentran que el tratamiento únicamente explica el $12.3 \%$ de la varianza de resultados si se usa la abstinencia como criterio. Estos hallazgos los resumen Edwards y col.(4), diciendo que " cuando se alarga el tiempo de seguimiento se tiene conciencia de que el tratamiento, a menudo, tiene una influencia pequeña y efímera en la evolución de la vida de los sujetos". Vaillant (21), explica e interpreta estos resultados teniendo en cuenta y asumiendo las características de la dependencia alcohólica como una enfermedad con una tendencia a la recaída que va más allá de la voluntad y motivación de los pacientes, y le lleva a plantear una nueva y más amplia visión del tratamiento, cuyas funciones serían similares a las que cumple en otras enfermedades crónicas como la diabetes y la hipertensión, es decir: funciones de estabilización, mantenimiento y resolución de crisis y recaídas.

Algunos datos de los efectos del tratamiento a largo plazo avalarían esta posición. Así, Emrick (26), en el estudio ya citado, concluía que los alcohólicos tratados tenían mayores tasas de mejoría que los no tratados; pero no mayores tasas de abstinencia y Moos y Finney (30) encontraban que si en lugar de las tasas de abstinencia se utiliza como criterio de evaluación la reducción del consumo, el peso del tratamiento en los resultados a largo plazo se multiplica por tres. También hay constancia de que la prolongación de los tratamientos en régimen de internamiento no aumenta las tasas de abstinencia en el seguimiento a medio plazo (31), y lo mismo ocurre con la mayor amplitud e intensidad de los tratamientos, que reducen las complicaciones y favorecen el funcionamiento de los pacientes pero no aumentan las tasas de abstinencia (32). Con los tratamientos farmacológicos los resultados son similares: las primeras revisiones de estudios de eficacia del disulfiram (33), ya encontraban que su acción principal más que aumentar las tasas de abstinencia, era reducir los días de consumo y la cantidad de alcohol consumido, y este hallazgo se repite en una revisión reciente de 24 estudios publicados en inglés entre 1967 y 1995 (34), y con los nuevos tratamientos farmacológicos, naltrexona y acamprosato, con los que el resultado más claro y más patente, y más si se prolonga el tiempo de seguimiento, es la reducción de alcohol consumido, de días de consumo, y de niveles de consumo tras la violación de la abstinencia $(35,36)$.

Podemos concluir que, en una buena parte de los dependientes del alcohol, los tratamientos son eficaces a corto plazo y mientras se están aplicando, disminuyendo su papel a medida que va pasando el tiempo desde el inicio del mismo y a medida que se prolonga el tiempo de seguimiento, y que sus efectos se manifiestan fundamentalmente en la reducción de los niveles de consumo y en la mejora del funcionamiento y calidad de vida de los pacientes. 


\section{DE LA PRESCRIPCION A LOS RESULTADOS}

La prescripción de abstinencia absoluta surge de una manera de entender la dependencia alcohólica que gira en torno a la ausencia de control sobre el consumo una vez iniciado éste. Desde este modelo, la prescripción de abstinencia constituye un instrumento terapéutico cuyo objetivo es la consecución de la abstinencia, entendida ésta como expresión de ausencia de enfermedad. De esta forma, la abstinencia, además de prescripción, se convierte en la meta del tratamiento y en criterio de evaluación de resultados. Es necesario que haya coherencia entre la prescripción y el modelo, ya que la aceptación de la prescripción por parte del paciente lleva implícita la aceptación de una manera de entender el problema y de unas estrategias para solucionarlo, que es el punto de partida para el cumplimiento y eficacia de los tratamientos.

Ahora bien, ¿si los tratamientos reducen los niveles de consumo y las tasas de recaída, a pesar del consumo, se debe cambiar la prescripción y buscar el control de la bebida más que la abstinencia?, ¿se puede cambiar la prescripción sin cambiar el modelo, no ya en la cabeza de los terapéutas sino, lo que es más importante, en la de los pacientes?, ¿todos los dependientes serán capaces de entender que si se les dice que beban poco no quiere decir que verdaderamente esté en su mano beber poco y que su problema no es únicamente un problema de cantidad?. A estas pregunta, impensables hace unos años no ya por Alcohólicos Anónimos y otros grupos de autoayuda, defensores de la abstinencia como objetivo y como modo de vida, sino por parte de los profesionales que se manejaban con el modelo médico de la enfermedad alcohólica, se está respondiendo ya en sentido afirmativo, sin profundizar demasiado en el asunto. En esta línea van algunas de las indicaciones que se están haciendo de los nuevos fármacos, como la recomendación de su uso los fines de semana, en dependientes del alcohol con patrones episódicos, con la información al paciente de que ayuda a reducir los niveles de consumo y le evita recaídas aunque consuma, lo que supone una prescripción de bebida controlada.

\subsection{La bebida controlada}

En los años 50, hasta el trabajo de Davies (16), el más citado sobre el tema, Lemere (37) había publicado un $3 \%$ sobre una muestra de 500 y Selzer y col.(38), un $16 \%$ de 83 a los 5 años. El trabajo de Davies se centró en 93 alcohólicos dados de alta en el Maudsley Hospital, de ellos 7, un 7.5\%, bebían sin problemas en un período de seguimiento de 7 a 11 años. Tasas en torno al 10\% se han publicado posteriormente $(10,6,17)$. Cuando se analiza la estabilidad del patrón de bebida controlada, aspecto básico de cara a la predicción y evolución a largo plazo, Vaillant (6) encuentra que mientras que el $86 \%$ de los que tenían el status de abstinencia absoluta seguían teniéndolo 15 años más tarde, únicamente un $36 \%$ de los que tenían el status de bebida controlada, lo mantenían al cabo de ese mismo período, lo que le permitía concluir que el retorno a patrones estables de bebida sin problemas entre alcohólicos crónicos es improbable.

Sin embargo, para los modelos psicológicos, la posibilidad de bebida controlada apoya sus ideas de la pérdida de control como un proceso adquirido y les lleva a plantearse la recaída, expresión conductual de la pérdida de control, como un fenómeno aprendido en el que intervienen múltiples factores cuyo control puede modificar el desenlace del inicio del consumo en una persona alcohólica, abriendo la puerta a la bebida controlada como objetivo terapéutico (39). Algunos factores como el papel de la prescripción y violación de la abstinencia o el papel de la atribución del control son determinantes en que se pueda dar la bebida controlada ó se termine produciendo la recaída tras el inicio del consumo. En este sentido se postula que los pacientes que se ven a si mismos como responsables del cambio en su conducta de bebida tienen más éxito en mantener el cambio, y que el caracterizar al alcoholismo como una enfermedad tiene un efecto negativo sobre los resultados, atribuyéndose la perdida de control al temor a perderlo cuando se inicia el consumo $(40,41)$.

En la aparición de la recaída se da mucha importancia al efecto de violación de la abstinencia, que se define como una reacción cognitiva afectiva ante un primer consumo tras un período de abstinencia (42). En el fondo, el efecto de violación de la abstinencia, el "priming" de las teorías actuales, es un artefacto común a ambos modelos, que al mismo tiempo que se señala y resalta su existencia, permite el diseño de estrategias para su control: farmacològicas en el caso de los modelos biológicos y técnicas psicológicas para el manejo de situaciones de alto riesgo en los programas de prevención de recaídas. Sin embargo, la experiencia clínica nos dice que no es un fenómeno ni nuclear ni universal en el proceso de recaída, que seguramente puede tener mucho peso en la decisión de consumir en pacientes con niveles de dependencia moderados, que necesitan niveles altos de consumo para que la falta de control se manifieste, con una estabilidad emocional y laboral buena y con una personalidad bien dotada, que les permite poner en marcha todo un conjunto de estrategias para no seguir bebiendo; pero que tiene poco peso en la evolución a largo plazo de los dependientes del alcohol que no cumplen los anteriores requisitos y donde la violación de la abstinencia es sinónimo de recaída. Un lugar de encuentro y una buena guía clínica, entre la prescripción de bebida controlada y de abstinencia absoluta, 
es el modelo dimensional de síndrome de dependencia, que plantea la abstinencia absoluta para la dependencia grave, pudiéndose plantear otros objetivos en la dependencia ligera y moderada (43).

\subsection{Papel de la prescripción en los resultados}

Algunos estudios nos dicen que lo que cuenta es el modelo transmitido y la congruencia entre la prescripción ligada al modelo y el criterio de evaluación de resultados. Así en la revisión de Monahan y Finney (27), que utilizaban como criterio de evaluación las tasas de abstinencia, se encuentra que aquellos programas de tratamiento con un objetivo expresado diferente a la abstinencia absoluta tienen tasas más bajas de abstinencia que aquellos con el objetivo de abstinencia. Nosotros, en un programa que prescribe la abstinencia, encontramos mayores tasas de recaída en aquellos que expresaban que su meta ideal del tratamiento era la bebida moderada (44). El papel del modelo transmitido queda también reflejado en el trabajo de Liskow y col.(45), que encuentran que la eficacia del disulfiram está no tanto en el cumplimiento del tratamiento como en lo que se transmite al paciente con respecto a lo que ocurrirá si dejan de tomarlo, y que está en línea con la interpretación que se hace en la actualidad del papel de los interdictores en la prevención de recaídas como disuasores: ayudando a decir no antes los impulsos de bebida, en el contexto de un modelo donde se transmite que el inicio del consumo supone el comienzo de la recaída.

Por lo tanto, si la prescripción de abstinencia lleva aparejado mayores tasas de abstinencia, el cambio de prescripción únicamente tendría sentido si se renuncia al objetivo de conseguir y mantener la abstinencia en función de la consecución de otros objetivos, lo que nos lleva a los programas de reducción de daños. Todo esto sin olvidar que la prescripción de abstinencia absoluta, aunque no consiga la abstinencia, puede llevar a reducir los niveles de consumo, por lo que no en todos aquellos que son incapaces de mantenerse abstinentes tendría sentido cambiar de prescripción.

\section{PROGRAMAS DE REDUCCION DE DAÑOS EN LA DEPENDENCIA ALCOHOLICA}

Desde la perspectiva de los problemas derivados del consumo de alcohol, todos los programas de intervención dirigidos a población de consumo de riesgo, de consumo excesivo y de problemas relacionados con el alcohol entrarían dentro de la categoría de programas de reducción de daños, actuando sobre los niveles de consumo y teniendo como objetivo no la abstinencia absoluta sino el situar el consumo por debajo de un umbral que se considera de riesgo, y que siguiendo patrones anglosajones se suele situar en $140 \mathrm{~g} / \mathrm{semana}$ para la mujer y $280 \mathrm{~g} / \mathrm{semana}$ para el hombre(46), y en nuestro medio el Programa de Actividades Preventivas y de Promoción de la Salud que se sigue en muchos Centros de Salud establece como límite $168 \mathrm{~g} / \mathrm{semana}$ para la mujer y el doble para el hombre (47).

En el caso de la dependencia alcohólica, las actuaciones para resolver las complicaciones asociadas al consumo, que son actuaciones de reducción de daños, se compaginan con la prescripción y el objetivo de la abstinencia absoluta como única manera de resolver la relación con el consumo. Sin embargo, en la clínica, cuando no se utilizan criterios estrictos para el mantenimiento en los programas de tratamiento, nos encontramos con un grupo de dependientes del alcohol con un nivel de deterioro somático y psicosocial importante, con una dependencia de más de 15 a 20 de años de evolución, que han seguido distintos tratamientos tanto en régimen de internamiento como ambulatorios, con múltiples y muy cortos períodos de abstinencia, y que su patrón de consumo se caracteriza por varios meses de altos niveles de consumo de alcohol (más de 150 o 200 g/día), alternando con cortos períodos de abstinencia (no más de cuatro o seis semanas), que coinciden en parte con lo que otros autores han denominado "enfermos en tratamiento de larga duración LTD" (48).

En este grupo de pacientes los objetivos terapéuticos van dirigidos a reducir y minimizar las consecuencias del consumo, reduciendo los niveles de éste, y manejando las situaciones clínicas ligadas a la intoxicación, asi como actuando sobre el medio sociofamiliar para reducir el impacto sobre éste de la permanencia y persistencia de los síntomas de la enfermedad. Lo mismo ocurre en los programas dirigidos a los dependientes del alcohol "sin hogar", donde se hace más hincapié en las actuaciones sobre las necesidades básicas de estas personas (alojamiento, comida, asistencia sanitaria) que sobre el consumo de alcohol (49). En estos casos el explicitar el cambio en la prioridad de los objetivos, unido a un cambio en la prescripción desde la abstinencia absoluta a la reducción del consumo podría facilitar la retención en los tratamientos y el abordaje de las complicaciones asociadas.

\subsection{Criterios de asignación}

Cuando los dependientes del alcohol entran en tratamiento ni las distintas tipologías utilizadas $(50,51)$, ni otros factores predicen la evolución a largo plazo (4, $52,53)$. El principal factor predictivo de evolución a largo plazo es la propia evolución a corto y medio plazo, sobre todo el criterio de abstinencia absoluta, aunque se estima entre un $3 \%$ y un $5 \%$ el riesgo anual de recaída entre aquellos que se mantienen 
abstinentes entre año y medio y dos años $(13,54)$. A mayor tiempo de abstinencia más probabilidades de mantenerse abstinente al año siguiente. Asi, en pacientes integrados en $A A$, estando abstinentes un año hay un $75 \%$ de probabilidades de mantenerse abstinente el siguiente año, si se esta abstinente dos años, las probabilidades se incrementan hasta el $85 \%$ y lo hacen hasta el $98 \%$ si se mantienen abstinentes durante cinco años (55).

Lo que está por delimitar es el tiempo que se requiere estar abstinente para asegurar la abstinencia a largo plazo. Algunos autores señalan que el funcionamiento a los 2 o 3 años predice el funcionamiento a los 8 o 10 año $(30,56$ ). Vaillant (6), estima en cinco a seis años el período que hay que tomar en consideración para que el status de bebida obtenido prediga con bastante fiabilidad el status de bebida a largo plazo.

Por lo tanto, para la asignación habría que tener en cuenta la respuesta al tratamiento y evolución durante un período no inferior a dos o tres años, que permita definir el status de consumo y la predicción del mismo a largo plazo y utilizar la latencia de recaída, tiempo que el paciente es capaz de mantenerse en abstinencia absoluta, como criterio de gravedad de la dependencia. En este sentido, si durante un periodo de tratamiento y seguimiento de más de dos años, el paciente es incapaz de mantenerse en abstinencia absoluta más de cuatro o seis semanas seguidas, estaría indicado un cambio de prescripción, pasando de la abstinencia absoluta a la reducción del consumo.

Una indicación clara son el grupo de los "sin hogar" donde entre un tercio y la mitad tienen una dependencia alcohólica $(56,57)$, que en la mayoría de los casos están sin tratar y a los pocos que acuden a tratamiento se les ofrecen programas terapéuticos generales, con prescripción de abstinencia absoluta y donde los resultados son muy malos, con tasas de abandono muy altas y tasas de abstinencia que no llegan al $10 \%(58,48)$.

\subsection{Características de los programas de reducción de daños}

El cambio de prescripción y la búsqueda de la reducción del consumo es únicamente un aspecto parcial de estos programas, ya que la reducción del consumo no implica obligatoriamente una mejoría de todos los problemas y enfermedades de los dependientes del alcohol. Existe una relación estrecha entre la reducción del consumo y la mejoría de las complicaciones somáticas $(30,59)$, pero no de una manera tan directa en el funcionamiento social y laboral, lo que implica la necesidad de intervenciones específicas sobre estas áreas, y que junto a las intervenciones sobre el consumo definen los programas de reducción de daños.
La hospitalización, frecuente y reiterada, seguirá siendo un recurso imprescindible tanto para resolver situaciones de crisis como para desintoxicar y establecer periodos de abstinencia prolongada que permitan el manejo de las complicaciones somáticas y psíquicas asociadas (48). En los casos más graves, la necesidad de alargar el ingreso va a requerir de dispositivos de estancia media y prolongada, asi como unidades residenciales para estancias permanentes.

Las técnicas psicoterapéuticas utilizadas en los programas de reducción de consumo en no dependientes (60), pueden ser igual de eficaces en los dependientes, pero no en programas de intervención breves y muy breves sino a largo plazo y de manera sostenida, asi como la utilización de distintos fármacos utilizados inicialmente para conseguir la abstinencia, que se han mostrado útiles en la reducción de los niveles de consumo. En este sentido, no se deben descartar las benzodiacepinas de vida media media o larga, cuya utilización de forma prolongada puede ayudar a reducir los niveles de consumo, aunque requieran estar atentos a la escalada de dosis o a la presencia de efectos paradójicos. Más útiles y con menos riesgos son aquellos fármacos sin capacidad adictiva y sin interacciones graves con el alcohol como son la buspirona, los inhibidores selectivos de la recaptación de la serotonina, el acamprosato y la naltrexona $(61,62)$.

Las intervenciones sobre las complicaciones y factores asociados al consumo, prioritarias en estos programas, van a suponer, como ha ocurrido en otras adicciones $(63,64,65)$, importantes cambios en los dispositivos asistenciales con incorporación de profesionales y redes sociales amplias. La retención en el tratamiento se convierte en el objetivo primordial y en indicador de calidad del programa y los buenos resultados se van a asociar a : fácil acceso al tratamiento, accesibilidad física del centro con horarios adecuados, accesibilidad de los miembros del equipo, calidez y permanencia del personal, coordinación con servicios sociales, diversidad de los servicios ofrecidos, orientación del tratamiento hacia el largo plazo, presencia de apoyo terapéutico, tratamiento focalizado en la rehabilitación, actitud del equipo terapéutico caracterizado por no presionar a los pacientes hacia la abstinencia y planeamiento del programa a plazo indefinido, flexibilidad e individualización del marco terapéutico (66).

\section{BIBLIOGRAFIA}

1. Lacoste JA. Reducción de daños y riesgos: una respuesta de salud pública a la infección por VIH entre usuarios de drogas. Adicciones 1995; 7:299-331.

2. Colom J. Los programas de reducción de riesgos. Adicciones 1997; 9:163-170. 
3. Markez I, Póo M. Nuevos modelos de intervención: disminución de riesgos y programas de mantenimiento. Psiquiatría Pública 1998; 10 (6):378-388.

4. Edwards G, Brown D, Oppenheimer E, Sheehan M, Taylor C. Duckitt A. Long-term outcome for patients with drinking problems: the search for predictors. Br J Addiction 1988; 83: 917-927.

5. Ojesjö L. Long-term outcome in alcohol abuse and alcoholism in the Lundby general population, sweden. Br J Addiction 1981; 76:391-400.

6. Vaillant G. A Long-term follow-up of male alcohol abuse. Arch Gen Psychiatry 1996; 53: 243-249.

7. Lundquist GAR. Alcohol dependence. Acta Psychiatr Scand 1973; 49:332-340.

8. Hyman MM. Alcohoolics 15 years later. Ann NY Acad Sci 1976; 273:613-623.

9. Vaillant GE, Clark W, Cyrus C, Milofsky ES, Kopp J, Wulsin VW, Mogielnicki NP. Prospective study of alcoholism treatment: eight-year follow-up. Am J Med 1983; 75:455-463.

10. Edwards G, Duckitt A, Oppenheimer E, Sheehan M, Taylor C. What happens to alcoholics ?. Lancet 1983; ii: 269-271.

11. O'Connor A, Daly J. A twenty year follow-up study. Br J Psychiatry 1985; 146:645-647.

12. Cross GM, Morgan CW, Mooney AJ, Martin CA, Rafter JA. Alcoholism treatment: a ten year follow-up study. Alc Clin Exp Res 1990; 14: 169-173.

13. Finney JW, Moos RH. The long-term course of treated alcoholism: I. Mortality, relapse and remission rates and comparison with community controls. J Stud Alcohol 1991; 52: 44-54.

14. Längle G, Mann K, Mindle G, Schied HW. Ten years afterthe post-treatment course of alcoholism. Eur Psychiatry 1993; 8: 95-100.

15. Shaw GK, Waller S, Letham CJ, Dunn G, Thomson AD. Alcoholism: A long-term follow-up study of participants in an alcohol treatment programme. Alc \& Alcoholism 1997; 32:527-533.

16. Davies DL. Normal drinking in recovered alcohol addicts. Q J Stud Alcohol 1962; 23: 94-104.

17. Gual A, LLigoña A, Colom J. Five-year outcome in alcohol dependence. A naturalistic study of 850 patients in Catalonia. Alc \& Alcoholism 1999; 34:183-192.

18. Alfano AM, Thurstin AH. Program evaluation research in ongoing alcoholism treatment: A summary of the Tuscaloosa VA Project. Int J Addiction 1989; 24:303-314.

19. Alford GS, Koehler RA, Leonard J. Alcoholics anonymous- narcotics anonymous model inpatient treatment of chemically dependent adolescents: a 2 year outcome study. J Stud Alcohol 1991; 52: 118-126.

20. Feuerlein W, Küfner $H$. A prospective multicentre study of in- patient treatment for alcoholics: 18 and 48 month follow-up (Munich evaluation for Alcoholism Treatment), MEAT. Eur Psychiatry Neurol Sci 1989; 239: 144-157.

21. Vaillant GE. What can long-term follow-up teach about relapse and prevention of relapse, in addiction ?. $\mathbf{B r} \mathbf{J}$ Addiction 1988; 83: 1147-1157.
22. Cuadrado P. Respuesta al tratamiento y evolución de la dependencia alcohólica. Seguimiento de 5-7 años. Archivos de Neurobiología (en prensa).

23. Cuadrado P, García R, Pata MD, Noval MJ. Respuesta de la dependencia alcohólica a un programa comunitario de tratamiento. Psiquiatría Pública 1998; 10: 162-168.

24. Cuadrado P, García R, Cadavid C, Lafarga C, Pata MD, Noval MJ. Tratamiento del alcoholismo en un centro de salud mental: datos de seguimiento a uno, tres y seis meses. Psiquiatría Pública 1993; 5: 176-184.

25. Cuadrado P, García R, Pata MD, Noval MJ. Tratamiento de alcohólicos en un programa comunitario. Resultados a los 6 meses, 1 año y 2 años. Adicciones 1995; 7: 169-177.

26. Emrick CD. A review of psychologically oriented treatment of alcoholism. II. The relative effectiveness of different treatment approaches and the effectiveness of treatment versus no treatment. J Stud Alcohol 1975; 36: 88-108.

27. Monahan SC, Finney JW. Explaining abstinence rates following treatment for alcohol abuse: a quantitative syntesis of patient, research design and treatment effects. Addiction 1996; 91: 787-805.

28. Costello RM. Alcoholism treatment and evaluations: in search methods II, collation of two year follow-up studies. Int J Addiction 1975; 10: 857-867.

29. Armor DJ, Polich JM, Stambul HB. Alcoholism and treatment. New York: John Wiley \& Sons Inc, 1978.

30. Moos RH, Finney JW, Cronkite RC. Alcoholism Treatment. New York: Oxford University Press, 1990: 17-35.

31. Feuerlein $W$, Küfner $H$. A prospective multicentre study of in- patient treatment for alcoholics: 18 and 48 month follow-up. Munich evaluation for Alcoholism Treatment, MEAT. Eur Arch Psychiatr Neurol Sci 1989; 239:144157.

32. Chick J, Ritson B, Connaughton J, Stewart A, Chick J. Advice versus extended treatment for alcoholism: a controlled study. Br J Addiction 1988; 83: 159-170.

33. Lundwall L, Baekeland F. Disulfiram treatment of alcoholism. J Nerv Ment Dis 1971; 153: 381-394.

34. Hughes JC, Cook CCH. The efficacy of disulfiram: a review of outcome studies. Addiction 1997; 92: 381395.

35. Soyka M. Profilaxis de las recaídas en el alcoholismo. Avances recientes y posibilidades futuras. CNS Drugs 1997; 7 (4): 313-327.

36. Volpicelli JR, Rhines KC, Rhines JS, Volpicelli LA, Alterman Al, O'Brien CP. Naltrexone and alcohol dependence. Role of subject compliance. Arch Gen Psychiatry 1997; 54: 737-742.

37. Lémere F. What happens to alcoholics. Am J Psychiatry 1953; 109: 674-676.

38. Selzer ML, Holloway HA. A follow-up of alcoholics commited to a state hospital. Q J Stud Alcohol 1957; 18 : 98-120.

39. Sobell MB, Sobell LC. Behavioral treatment of alcohol problems. Individualized therapy and controlled drinking. New York: Plenum Press, 1978. 
40. Sanchez-Craig M. Brief didactic treatment for alcohol and drug-related problems: an approach based on client choice. Br J Addiction 1990; 85: 169-177.

41. Miller WR, Westenberg VS, Harris RJ, Tonigan JS. What predicts relapse?. Prospective testing of antecedents models. Addiction 1996; 91 (Suppl.):S155-S171.

42. Marlatt $A$. La prevención de recaídas en las conductas adictivas: un enfoque de tratamiento cognitivo conductual. En: Casas M, Gossop M (eds) recaída y prevención de recaídas. Sitges: Ediciones en Neurociencias, 1993.

43. Edwards G. The practical business of treatment-15. The alcoholism treatment service at the Maudsley Hospital, London. Br J Addiction 1991; 86:143-150.

44. García R, Cuadrado P, Noval MJ, Pata MD. Factores implicados en la recaída en un programa ambulatorio de dependencia del alcohol. Comunicación a las XIX Jornadas Nacionales de Socidrogalcohol. Santa Cruz de Tenerife, 1991

45. Liskow B, Nickel E, Linley N. Alcoholics' attituds toward and experiences with disulfiram. Am J Drud Alcohol Abuse 1990; 16:147-160.

46. National Health and Medical Research Council. Is there a safe level of daily consumption of alcohol for men and women: Recomendations regarding responsible drinking behaviour. Camberra: Australian Government Publishing Service, 1987.

47. Cuadrado P, Martínez JJ, Picatoste J. El profesional de Atención Primaria de Salud ante los problemas derivados del consumo de alcohol. Madrid: Plan Nacional sobre Drogas, Ministerio de Justicia e Interior, Madrid, 1994

48. Monrás M, García S, Torres M, Gual A, Ortega L. El ingreso hospitalario en el tratamiento de los alcohólicos cronificados. Adicciones 1998; 10: 151-160.

49. Wright JD, Devine JA. Factors that interact with treatment to predict outcomes in substance abuse programs for the homeless. J Addictive Diseases 1995; 14: 169-181.

50. Babor TS, Dolinsky ZG, Meyer RE, Hesselbrock M, Hofmann M, Tennen H. Types of alcoholics: concurrent and predictive validity of some common classification schemes. Br J Addiction 1992; 87: 1415-1431.
51. Yates WR, Booth BM, Read DA, Brown K, Martenson BJ. Descriptive and predictive validity of a high-risk alcoholism relapse model. J Stud Alcohol 1993; 54:641-651.

52. Cuadrado P. Evolución de la dependencia alcohólica en tratamiento. Factores predictivos en un seguimiento de 5 a 7 años. Adicciones 1998; 10: 335-341.

53. Vaillant GE. The natural history of alcoholism revisited. Cambridge, Mass: Harvard University Press, 1995.

54. Jin H, Rourke SB, Patterson TL, Taylor MJ, Grant I. Predictors of relapse in long-term abstinent alcoholics. J Stud Alcohol 1998; 59: 640-646.

55. Miller NS, Gold MS (eds.). Alcohol. New York: Plenum Medical Book Company, 1991: 131-159.

56. Rico P, Vega LS, Aranguren L. Trastornos psiquiátricos en transeúntes: un estudio epidemiológico en Aranjuez. Rev Asoc Esp Neuropsiq 1994; XIV: 633-649.

57. Kasprow WJ, Rosenheck R. Substance use and psychiatric problems of homeless native american veterans. Psychiatric Services 1998; 49: 345-350.

58. Feuerlein W. Treatment of particular populations of alcoholics. Alcoología 1993; 5:153-158.

59. Edwards G y col. Alcohol y Salud Pública. Barcelona: Prous Science, 1997:38-67.

60. Bien TH, Miller WR, Tonigan JS. Brief interventions for alcohol problems: a review. Addiction 1993; 88: 315336.

61. Sinclair JD. The feasibility of effective psychopharmacological treatments of alcoholism. Br J Addiction 1987; 82: 1213-1223.

62. Litten RZ, Allen J, Fertig J. Pharmacotherapies for alcohol problems: A review of research with focus on developments since 1991. Alc Clin Exp Res 1996; 20:859-876.

63. McLellan AT, Arndt IO, Metzger DS, Woody GE, O’Brien CP. The effect of psychosocial services in substance abuse treatment. JAMA 1993; 269: 1953-1959.

64. O'Hara PA, Newcombe R, Matthews A, Bunning EC, Drucker $E$. La reducción de los daños relacionados con las drogas. Barcelona: Grupo Igia, 1995.

65. Igia Group. Programas de reducción de riesgos. Barcelona: Mimeo ed., 1996

66. Del Río M. Farmacoterapia de sustitución en la dependencia a opiaceos. Monografías en Psiquiatría 1998; $\mathrm{x}: 3-12$ 